\title{
Can Be Done Better for the Diagnosis of Non-Differentiated Diseases in Community Hospital?
}

\section{Xurui Li',2, Zhangshun Shen², Chang Lv², Huiling Zang², Junna Zhang1, Qian Zhao², Hui Guo², Jianguo $\mathbf{L i}^{2}$}

${ }^{1}$ General Practice Department, Hebei General Hospital, Shijiazhuang, China

${ }^{2}$ Department of Emergency, Hebei General Hospital, Shijiazhuang, China

Email: lijg65@163.com

How to cite this paper: Li, X.R., Shen, Z.S., Lv, C., Zang, H.L., Zhang, J.N., Zhao, Q., Guo, H. and Li, J.G. (2021) Can Be Done Better for the Diagnosis of Non-Differentiated Diseases in Community Hospital?. Case Reports in Clinical Medicine, 10, 323-329.

https://doi.org/10.4236/crcm.2021.1010041

Received: September 22, 2021

Accepted: October 22, 2021

Published: October 25, 2021

Copyright $\odot 2021$ by author(s) and Scientific Research Publishing Inc. This work is licensed under the Creative Commons Attribution International License (CC BY 4.0).

http://creativecommons.org/licenses/by/4.0/

\begin{abstract}
General Practice is a comprehensive subject with a wide spectrum of diseases. It is challenging to make a more correct diagnosis timely and reasonable treatment with the least instrument examination for the diagnosis of medically unexplained physical symptoms (MUPS). This article reviews the case report of a patient who presented to a community hospital with a one-month history of constant diffuse abdominal pain and underwent eight weeks of examinations, finally experienced deterioration of their condition. Based on this case, this article discusses the problems in the diagnosis of abdominal pain in a community hospital, and according to the clinical thinking mode and checklist of abdominal pain, the diagnostic process of abdominal pain can be improved, and the diagnosis of non-differentiated diseases can be done better, the harm caused by delayed diagnosis may be reduced.
\end{abstract}

\section{Keywords}

Medically Unexplained Physical Symptoms, General Practice Thinking, Checklist, Abdominal Pain

\section{Introduction}

General practitioners often get a more appropriate diagnosis with the least instrument examination for the diagnosis of medically unexplained physical symptoms (MUPS), which may also produce inertial thinking and get into confusion. With the development of general medicine, the discussion of thinking mode of general medicine is increasing day by day [1] [2]. How to make an effective diagnosis has always been an issue. The recent article entitled "Led Astray" re- 
counts a meaningful and complete case of abdominal pain [3]. In this case, the patient presented to a community hospital with a one-month history of constant diffuse abdominal pain. After eight weeks of examinations and deterioration of their condition, and diagnosis was delayed to threaten the patient's life. Whether a more effective mode of thinking can be used to solve this problem is worth considering. This article reviews this case, and discusses a clinical symptom-oriented modular diagnosis scheme to improve the diagnostic process of abdominal pain [4].

\section{Simple Review of the Case Report [3]}

A 34-year-old man presented to a community hospital in Vancouver, Canada, with a 1-month history of constant diffuse abdominal pain, fatigue, and anorexia associated with a weight loss of $2.7 \mathrm{~kg}$ but no fevers or night sweats. He reported constipation but no diarrhea, melena, or hematochezia. His medical history and travel history were unremarkable. He worked at a family-run business and lived with his wife and his parents. He did not use medications leading to his recent paint.

In this case, anemia and liver dysfunction were identified as prominent manifestations after the relevant laboratory examination. At the same time, computed tomography (CT) of the abdomen revealed thickening of the rectum and sigmoid colon near the hepatic flexure. This prompted an upper and lower endoscopy and endoscopic pathological examinations, but no positive findings were found. The initial diagnosis was thalassemia, but thalassemia could not explain the abdominal pain. During the next six weeks, the patient visited the emergency department for ongoing abdominal pain three times, confirming that a clear diagnosis was not achieved. During the seventh week, the patient developed a headache and fever in addition to the abdominal pain that had not been alleviated. Further tests and examinations were performed, including serologic testing, lumbar puncture, blood and urine cultures, chest radiography, magnetic resonance imaging of the head and spine, and capsule endoscopy, but no unifying diagnosis was identified. The patient developed epilepsy in the eighth week and was transferred to the intensive care unit with tracheal intubation. Finally, it was found that the serum-free protoporphyrin level was increased, and blood lead level was significantly elevated. Consequently, the correct diagnosis of lead poisoning was identified. On this basis, the patient was again asked about his history, and it was clear that the patient had been using lead-containing opium, which was the cause of poisoning.

As the authors described, the differential diagnosis of subacute, diffuse abdominal pain in a young man is broad [5]. Practitioners need to inquire further to obtain a thorough medical history, physical examinations, and laboratory examination for these clinical problems.

\section{Earlier Diagnosis Using a Clinical Thinking Checklist}

There are many potential causes of abdominal pain, including external abdominal injuries, chest, and systemic disease. These may involve a range of medical 
disciplines, including internal medicine, surgery, obstetrics, and gynecology, to accurately diagnose [3]. We propose that a clinical thinking checklist may have improved the diagnostic process in the present case. This scheme (Table 1) was based on holistic medicine for abdominal pain and formed the diagnosis model [4]. The top level of the modular scheme comprised five key directions according to the principle of anatomical positions (local, adjacent, systemic, mental, and gynecology). The second and third levels of the protocol applied anatomical concepts to define subcategories. The fourth level of the protocol aligned the diseases that cause abdominal pain based on the common diseases diagnosed by clinical decision-making and the principle of most common illnesses. This modular differential diagnosis scheme for abdominal pain is in line with the actual clinical setting and the differential diagnosis thinking concept. The diagnosis process of disease needs a clear diagnosis direction. The premise of diagnosis is the correct direction. The checklist focuses on guiding the clinical thinking direction of abdominal pain diagnosis. It is proposed that this clinical thinking checklist would help to prevent missed and erroneous diagnoses.

Table 1. Schematic checklist of the potential causes of abdominal pain [4].

\begin{tabular}{|c|c|c|c|}
\hline & $\begin{array}{l}\text { tomical } \\
\text { itioning }\end{array}$ & $\begin{array}{c}\text { Etiological } \\
\text { classification }\end{array}$ & Common diseases \\
\hline \multirow{5}{*}{\multicolumn{2}{|c|}{$\begin{array}{l}\text { Local } \\
\text { organ } \\
\text { disease }\end{array}$}} & Inflammation & $\begin{array}{l}\text { Gastritis, enteritis, pancreatitis, hemorrhagic } \\
\text { necrotizing enteritis, cholecystitis, suppurative } \\
\text { obstructive cholecystitis, urinary } \\
\text { tract infection, etc. }\end{array}$ \\
\hline & & Organ rupture & Liver or splenic rupture, perforation ulcer, etc. \\
\hline & & $\begin{array}{l}\text { Cavity organ } \\
\text { obstruction }\end{array}$ & $\begin{array}{l}\text { Intestinal obstruction, biliary calculi, biliary } \\
\text { ascariasis, renal ureteral calculi obstruction, etc. }\end{array}$ \\
\hline & & Reversal of organs & Volvulus, mesenteric or omentum torsion, etc. \\
\hline & & $\begin{array}{l}\text { Intraperitoneal } \\
\text { vascular lesions }\end{array}$ & $\begin{array}{l}\text { Mesenteric artery, splenic, renal embolism, } \\
\text { abdominal aortic dissection aneurysm, etc. }\end{array}$ \\
\hline & Napes & $\begin{array}{l}\text { Inflammation or } \\
\text { nerves }\end{array}$ & $\begin{array}{l}\text { Abdominal wall contusion, abscess } \\
\text { and herpes zoster, etc. }\end{array}$ \\
\hline \multicolumn{2}{|c|}{ Adjacent organ diseases } & $\begin{array}{l}\text { Spine, heart, and } \\
\text { lung diseases }\end{array}$ & $\begin{array}{l}\text { Angina pectoris, myocardial infarction, acute } \\
\text { pericarditis, pneumonia, pulmonary infarction, } \\
\text { pleurisy, hiatal hernia, thoracic tuberculosis } \\
\text { or tumor, etc. }\end{array}$ \\
\hline \multicolumn{3}{|c|}{ Systemic diseases } & $\begin{array}{l}\text { Abdominal Henoch-Schonlein purpura, } \\
\text { abdominal epilepsy, hematoporphyria, } \\
\text { erythematosus, poisoning, etc. }\end{array}$ \\
\hline \multicolumn{3}{|c|}{ Mental disorders } & Abdominal neurosis. \\
\hline \multicolumn{3}{|c|}{ Gynecology } & $\begin{array}{l}\text { Ectopic pregnancy and ovarian rupture, } \\
\text { ovarian cyst torsion, pelvic inflammation, } \\
\text { ovarian hemorrhage, etc. }\end{array}$ \\
\hline
\end{tabular}


According to the checklist, the five key directions (local, adjacent, systemic, mental, and gynecology) were screened one by one.

1) Local: Physical examination confirmed the abdominal pain was not caused by abdominal wall lesions. The abdominal examination revealed mild tenderness in the lower left quadrant, with no rebound tenderness, guarding, ascites, masses, or organomegaly. Bowel sounds were normal. There was no palpable lymphadenopathy. The hemoglobin level was $7.7 \mathrm{~g}$ per deciliter, and hematocrit was $25 \%$. CT of the abdomen revealed thickening of the rectum and sigmoid colon near the hepatic flexure, with normal-appearing terminal ileum and no lymphadenopathy. These findings exclude the diagnosis of abdominal pain caused by intraperitoneal intestinal rupture bleeding, intestinal torsion, and psycho-neuropathic abdominal pain, but vascular diseases and tumors could not be ruled out. Although acute vascular diseases generally cause severe pain, chronic vascular diseases can cause diffuse abdominal pain, debilitating diseases, and anemia.

2) Adjacent: For potential adjacent organ diseases, subacute and diffuse abdominal pain is a rare cardiopulmonary problem, but it does need to be considered. The patient's breathing was stable, without cough or expectoration. Abdominal pain was not related to breathing and exercise. It seems that pulmonary and cardiogenic pain was not considered, such as angina pectoris, myocardial infarction, acute pericarditis, pneumonia, pulmonary infarction, or pleurisy. Also, regardless of esophageal hiatal hernia, thoracic tuberculosis or tumor from CT examination were not described.

3) Systemic disease:

The patient's diagnosis was limited to chronic vascular diseases, tumors, and systemic disease. Considering the abdominal pain, anemia and abnormal liver function, according to the diagnosis direction of the checklist guidance, we need to be considered that whether there are systemic factors caused by abdominal pain. There are many potential reasons for abdominal pain caused by systemic factors, such as diabetic acidosis, abdominal Henoch-Schonlein purpura, abdominal epilepsy, uremia, hematoporphyria, systemic lupus erythematosus, and poisoning (lead, thallium).

4) Gynecology: The patient was a 34-year-old male, so gynecological diseases were ruled out.

5) Mental: Except for organic disease, the patient had anemia, liver abnormalities, so mental diseases were ruled out.

\section{Discussion}

General practitioners often get a more appropriate diagnosis with the least instrument examination for the diagnosis of MUPS. It is challenging to make a more correct diagnosis timely and reasonable treatment with the least instrument examination in a short time. Abdominal pain is a common undifferentiated disease among general practitioners.

There are many potential causes of abdominal pain, many of which fall under 
the purview of a range of medical disciplines. The differential diagnosis of abdominal pain presents hundreds of possible causes, making it easy to miss a diagnosis or even misdiagnose, and a delayed diagnosis can threaten the life of a patient. Abdominal pain is difficult to diagnose and has a high misdiagnosis and missed diagnosis rate. Birgisdottir et al. also reported abdominal pain caused by acute porphyria [6]. Two cases of abdominal Henoch-Schonlein purpura with abdominal pain were reported, and surgical treatment was performed. One patient died due to a postoperative intestinal fistula [7]. Lead poisoning is a diagnosis that is often overlooked. A case of abdominal pain caused by lead poisoning was misdiagnosed as nonspecific abdominal pain and acute porphyria [8]. In this case of non-iron-deficiency anemia, the typical clinical features of abdominal pain and weight loss were nonspecific, and the patient did not disclose his habits. The doctor did not get a heightened awareness of the possibility of some habits as a potential source. It is also difficult to think of lead poisoning and to be aware that opium contains lead. It is mentioned that incomplete medical history is an important cause of misdiagnosis. Most patients do not hide their medical history, but clinical thinking tells us that the patient was an individual who chose to withhold information, so other patients will do this in the future. Although patients hide their medical history (history of drugs and psychotropic drugs), referring to the checklist can avoid this situation.

From a clinical thinking point of view, we should consider the uncertainty and probability of a given diagnosis. Therefore, clinical reasoning based on these manifestations should also prompt consideration of other potential diagnoses that may be more likely. Over the next six weeks, the patient returned to the hospital emergency department three times because of continuing abdominal pain. The corresponding clinical decision should carry out further non-invasive examinations to except for the possibility of other systemic diseases. Lead poisoning only needs to be drawn for blood testing. Through early detection of elevated serum protoporphyrin levels, blood lead levels will increase significantly. There is no need to perform invasive examinations such as endoscopy early, which increases the patient's pain and prolongs the diagnosis time. If this had been performed, it might have been possible to identify elevated levels of serum-free protoporphyrin and blood lead earlier in the diagnostic process. If a cause was not found after screening for systemic factors, it might have endoscopic examination to preclude tumor and chronic vascular disease. Even if invasive examinations are conducted, and the results cannot be explained in the overall thinking model and monistic perspective, a comprehensive screening should be conducted according to the checklist again.

During differential diagnosis for abdominal pain, physicians are required to be aware of hundreds of potential causes. "I don't know" is perhaps the most important three-word sentence in medicine [9]. Physicians deal with uncertainty in medicine, and "I don't know" contributes to the uncertainty in the clinical thinking process, which guides physicians' actions in clinical practice [10]. It is 
challenging for doctors to make the correct diagnosis with a low number of examinations, so a checklist can ensure medical safety. The checklist is not just the direction of diagnosis we need to pay attention to, but also to develop a mode of thinking.

In conclusion, abdominal pain masks atypical diseases or rare diseases that are more prone to misdiagnosis and missed diagnosis [11]. If the current diagnosis cannot be explained, screening cause of disease one by one according to the checklist should consider poisoning earlier in the clinical course. Then, the availability, accuracy, and relatively low cost of serum testing should be performed promptly, which could get the diagnosis to be faster for MUPS in Community medical institutions.

\section{Acknowledgements}

We would like to acknowledge the hard and dedicated work of all the staff that implemented the intervention and evaluation components of the study.

\section{Competing Interests}

The authors declare that they have no competing interests.

\section{References}

[1] Qian, Y.M., Guo, J., Qian, F.H., et al. (2015) Study on the Training of Clinical Divergent Thinking in General Practitioner. Shanghai Medical \& Pharmaceutical Journal, 2015, 62-64.

[2] Tian, S.L., Yin, L., Tan, W., et al. (2017) Introduction to General Resident Standardization Training in Clinical Thinking Ability. China Continuing Medical Education, 2017, 3-4.

[3] Young, S., Chen, L., Palatnick, W., et al. (2020) Led Astray. The New England Journal of Medicine, 383, 578-583. https://doi.org/10.1056/NEJMcps1900799

[4] Pan, H.M., Li, H.L., Shen, Z.S., et al. (2021) Observation of the Effectiveness of a Diagnostic Model for Acute Abdominal Pain Based on the Etiology Checklist and Process Thinking. Risk Management and Healthcare Policy, 14, 835-845. https://doi.org/10.2147/RMHP.S295142

[5] Zhang, Y.q., Wang, Y.x. and Li, X.y., et al. (2016) Analysis on the Regularity of Emergency Disease Spectrum of 106,551 Patients. Chinese Journal of Critical Care Medicine, 36, 254-257.

[6] Birgisdottir, B.T., Asgeirsson, H., Arnardottir, S., et al. (2010) Acute Abdominal Pain Caused by Acute Intermittent Porphyria-Case Report and Review of the Literature. Laeknabladid, 96, 413-418. https://doi.org/10.17992/lbl.2010.06.301

[7] Meng, Q.y. (2018) "Ghost” in Differential Diagnosis of Abdominal Pain-Abdominal Purpura. Clinical Misdiagnosis \& Mistherapy, 31, 67-69.

[8] Tsai, M.T., Huang, S.Y. and Cheng, S.Y. (2017) Lead Poisoning Can Be Easily Misdiagnosed as Acute Porphyria and Nonspecific Abdominal Pain. Case Reports in Emergency Medicine, 2017, Article ID: 9050713. https://doi.org/10.1155/2017/9050713

[9] Morgan, M. (2019) Those Three Little Words. The BMJ, 367, 15918. 
https://doi.org/10.1136/bmj.15918

[10] Li, J.G., Lv, C. and Zhao, Q. (2019) Discussion on the Uncertainty of Clinical Thinking and Its Coping Strategies. Medicine \& Philosophy, 40, 14-17.

[11] Zhang, H.Y., Fan, Z. and Ma, F.Q. (2010) Clinical Pathological Analysis of Sudden Cardiac Death with Abdominal Pain as the Performance in 5 Patients. Modern Preventive Medicine, 37, 2926-2927. 